\title{
Relativistic strange stars with anisotropy and B-parameter in pseudo spheroidal space time
}

\author{
P. K. Chattopadhyay ${ }^{1}$ and B. C. Paul ${ }^{2}$ \\ ${ }^{1}$ Department of Physics, Alipurduar College, Jalpaiguri, 736122, West Bengal, India \\ email:pkl_760rediffmail.com \\ ${ }^{2}$ Department of Physics, North Bengal University, Siliguri, 734013, West Bengal, India \\ email: bcpaul@iucaa.ernet.in
}

\begin{abstract}
A class of compact cold stars in the presence of strange matter is obtained for a pseudo-spheroidal geometry. Considering the strange matter equation of state $p=\frac{1}{3}(\rho-4 B)$, with pressure anisotropy described by Vaidya-Tikekar metric, we determine the parameter $B$ both inside and on the surface of the star for different values of anisotropy parameter $\alpha$. In the anisotropic case, we note that a stable model of a compact star may be realized.
\end{abstract}

Keywords. equation of state

\section{Introduction}

In relativistic astrophysics, the estimated masses and radii for many compact objects are not compatible with the standard neutron star models. To understand the behaviour of observed physical features of such compact objects, it has been predicted that strange quark matter may be a useful approach ( $\mathrm{Li}$ et al. 1995; Bombaci 1997). In the case of compact objects an alternative approach was adopted (Mukherjee et al. 1997) to study the variation of pressure $p$ and density $\rho$ inside the stars based on the model (Vaidya \& Tikekar 1982; Tikekar \& Thomas 1999) with specific ansatz $e^{2 \mu}=\frac{1+\lambda r^{2} / R^{2}}{1+r^{2} / R^{2}}(\lambda=$ spheroidicity and $R=$ Curvature parameter), prescribing 3-pseudo spheroidal geometries for the 3 -space of the interior space-time of the star. The equation of state for the strange quark matter from Kapusta (1994) is:

$$
p=\frac{1}{3}(\rho-4 B)
$$

where $B$ is referred to as the Bag constant. The total energy density is $\rho=\rho_{q}+B$ and total pressure is $p=p_{q}-B$.

\section{Anisotropic compact star model}

The metric of a spherically symmetric, static, cold compact star in equilibrium is represented by,

$$
d s^{2}=-e^{2 \nu(r)} d t^{2}+e^{2 \mu(r)} d r^{2}+r^{2}\left(d \theta^{2}+\sin ^{2} \theta d \phi^{2}\right)
$$

where $\nu(r)$ and $\mu(r)$ are the two unknown metric functions. The energy-momentum tensor for the interior matter content of the ultra compact star with anisotropic fluid pressure is given by, $T_{i j}=\operatorname{diag}\left(-\rho, p_{r}, p_{\perp}, p_{\perp}\right)$, where $\rho, p_{r}$ and $p_{\perp}$ are the energy-density, radial pressure and tangential pressure respectively. In this model, pressure anisotropy 
(Tikekar \& Thomas 1999) is defined as $\Delta=p_{\perp}-p_{r}$, which depends on metric functions $\mu(r)$ and $\nu(r)$. The Einstein field equation is,

$$
\mathbf{R}_{i j}-\frac{1}{2} g_{i j} \mathbf{R}=T_{i j}
$$

where $\mathbf{R}_{i j}$ is Ricci tensor and $\mathbf{R}$ is the Ricci scalar. Using Eq. (2.1) in Eq. (2.2), we obtain the following equations:

$$
\begin{gathered}
\rho=\frac{\left(1-e^{-2 \mu}\right)}{r^{2}}+\frac{2 \mu^{\prime} e^{-2 \mu}}{r} \\
p_{r}=\frac{2 \nu^{\prime} e^{-2 \mu}}{r}-\frac{\left(1-e^{-2 \mu}\right)}{r^{2}} \\
\Delta e^{2 \mu}=\left[\nu^{\prime \prime}+\nu^{\prime 2}-\nu^{\prime} \mu^{\prime}-\frac{\nu^{\prime}}{r}-\frac{\mu^{\prime}}{r}-\frac{\left(1-e^{-2 \mu}\right)}{r^{2}}\right] .
\end{gathered}
$$

To simplify we choose the anisotropy parameter $\Delta$ defined above as $\alpha=\frac{\Delta R^{2}\left(1-\lambda+\lambda x^{2}\right)^{2}}{\lambda^{2}\left(x^{2}-1\right)}$ so that the regularity in $\operatorname{pressure}(\mathrm{p})$ and $\operatorname{density}(\rho)$ at the center of the star is ensured. Now using Eqs. (2.4), (2.5) and the ansatz $e^{2 \mu}=\frac{1+\lambda r^{2} / R^{2}}{1+r^{2} / R^{2}}$, one obtains a second order differential equation in 'z' (Mukherjee et al. 1997) given by,

$$
\left(1-z^{2}\right) \Psi_{z z}+z \Psi_{z}+\left(\beta^{2}-1\right) \Psi=0
$$

where $|\beta|^{2}=(2-\lambda+\lambda \alpha), z=\sqrt{\lambda /(\lambda-1)} x$ with $x^{2}=1+\frac{r^{2}}{R^{2}}$ and $\Psi=e^{\nu(r)}$. General solutions of Eq. (2.6) are given below:

Case(i) Here $\beta(=\sqrt{2-\lambda(1-\alpha)})$ is positive for the values of $\lambda$ and $\alpha$. The solution is

$$
\Psi=C\left[\beta \sqrt{z^{2}-1} \cosh (\beta \eta)-z \sinh (\beta \eta)\right]+D\left[\beta \sqrt{z^{2}-1} \sinh (\beta \eta)-z \cosh (\beta \eta)\right]
$$

Case(ii) Here $\beta(=\sqrt{\lambda(1-\alpha)-2})$ is positive for the values of $\lambda$ and $\alpha$. The solution is

$$
\Psi=C\left[\beta \sqrt{z^{2}-1} \cos (\beta \eta)-z \sin (\beta \eta)\right]+D\left[\beta \sqrt{z^{2}-1} \sin (\beta \eta)-z \cos (\beta \eta)\right]
$$

where $z=\cosh (\eta) . C$ and $D$ are two unknown constants. For an isotropic case $(\alpha=0)$, it reduces to the solutions obtained by Tikekar \& Jotania (2005).

\section{Discussion}

The variation of energy density and pressure inside a compact stellar object can be understood qualitatively in this model from Eqs. (2.3) and (2.4). The energy density $\rho$ and radial pressure $p_{r}$ become:

$$
\begin{aligned}
& \rho=\frac{1}{R^{2}\left(z^{2}-1\right)}\left[1+\frac{2}{(\lambda-1)\left(z^{2}-1\right)}\right] \\
& p_{r}=-\frac{1}{R^{2}\left(z^{2}-1\right)}\left[1-\frac{2 z}{(\lambda-1)} \frac{\Psi_{z}}{\Psi}\right]
\end{aligned}
$$

Using the expression of $\rho$ and $p_{r}$, the parameter $B$ may now be evaluated from Eq. (1.1). Here the unit of $B$ is $M e v / \mathrm{fm}^{3}$ when $R$ is expressed in $\mathrm{km}$. The following conditions may be imposed for a compact star: (i) At the boundary of the star Schwarzchild's exterior solution is matched with the interior solution i.e.

$$
e^{2 \nu(r=b)}=e^{-2 \mu(r=b)}=\left(1-\frac{2 M}{b}\right)
$$


(ii) Inside the star radial pressure $p_{r}>0$, which leads to an inequality $\frac{\Psi_{z}}{\Psi}<\frac{(\lambda-1)}{2 z}$. However at $r=b$ (defines the boundary of the star), the radial pressure $p_{r}=0$, which yields $\frac{\Psi_{z}\left(z_{b}\right)}{\Psi\left(z_{b}\right)}=\frac{(\lambda-1)}{2 z_{b}}$, where $z_{b}^{2}=\frac{\lambda\left(1+b^{2} / R^{2}\right)}{(\lambda-1)}$. Now $R$ can be evaluated using Eq. (3.2) for specific configuration of the compact object. To determine the role of anisotropy on $B$, we determine the two unknown parameters $\mathrm{C}$ and $\mathrm{D}$ that appear in Eqs. (2.7) and (2.8) from matching condition. Once $\mathrm{C}, \mathrm{D}$ and $R$ are known, the parameter $B$ can be determined using Eq. (3.1) at different points in the star for different $\alpha$ and $\lambda$.

Case I: The data for X-ray pulsar Her X-1 are mass $M=0.88 M_{\odot}$, and radius $b=7.7 \mathrm{~km}$, so that compactness $u=M / b=0.1686 . R=3.26376 \mathrm{~km}$ for $\lambda=1.6$. Inside the star the value of $B$ decreases from center to surface for a particular choice of $\alpha$ and at the surface it attains a constant value $B_{b}$ independent of the anisotropy parameter $\alpha$. At the center $B_{0}=86.2771 \mathrm{Mev} / \mathrm{fm}^{3}$ for $\alpha=0, B_{0}=143.067 \mathrm{Mev} / \mathrm{fm}^{3}$ for $\alpha=0.15$ and $B_{b}=4.81202 \mathrm{Mev} / \mathrm{fm}^{3}$.

Case II: The data for SAX J 1808.4-3658 are mass $M=1.323 M_{\odot}$ and radius $b=$ $6.55 \mathrm{Km}$. so that $u=0.2979$ (Tikekar \& Jotania 2005). Here $R=4.2668 \mathrm{Km}$ for $\lambda=3.1$. $B_{0}=-2.55601 \mathrm{Mev} / \mathrm{fm}^{3}$ for $\alpha=0, B_{0}=125.271 \mathrm{Mev} / \mathrm{fm}^{3}$ for $\alpha=0.3$ and $B_{b}=$ $7.09937 \mathrm{Mev} / \mathrm{fm}^{3}$. From the analysis for both cases it appears that $B$ parameter picks up smaller values for isotropic case and increases in the presence of anisotropy. At the center of the star the value of $B_{0}$ is found to increase almost linearly with an increase of anisotropy $\alpha$.

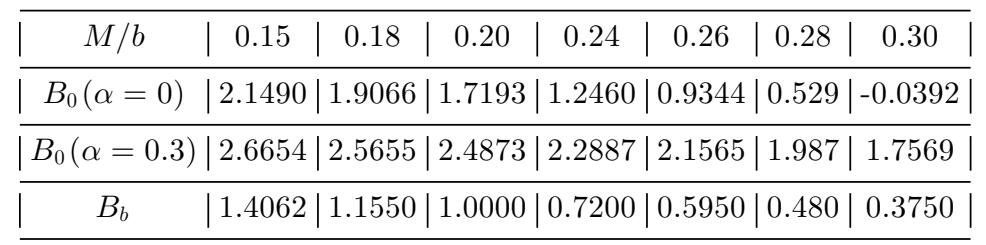

Table 1. Variation of parameter $B$ at the center $\left(B_{0}\right)$ and $\operatorname{surface}\left(B_{b}\right)$ in unit of $\frac{3 * 10^{4}}{R^{2}} \mathrm{Mev} / \mathrm{fm}^{3}$ with compactness factor $(M / b)$ for $\lambda=5$.

From Table 1 it is evident that at the surface of the star $B_{b}$ decreases with an increase of compactness $(M / b)$ for fixed $\alpha$. It is also noted that $B$ becomes negative near the centre of the star implying the core-region of such configurations to be repulsive. Thus the radial dependence of $B$ parameter in presence of isotropic and anisotropic strange matter are different throughout the interior of the star although at the boundary it attains a definite value.

\section{References}

Bombaci, I. 1997, Phys. Rev. C, 55, 1587

Kapusta, J. 1994, Finite-Temperature Field Theory (Cambridge University Press)

Li, X. D., Dai, Z. G., \& Wang, Z. R. 1995, Astron. Astrophysics, 303, L1

Mukherjee, S., Paul, B. C. \& Dadhich, N. 1997, Class. Quantum Grav. 14, 3475

Tikekar, R. \& Thomas, V. O. 1999, Pramana J. Phys. 52, 237

Tikekar, R. \& Jotania, K. 2005, Int. J. Mod. Phys. D, Vol. 14, No. 6 1037-1048

Vaidya, P. C. \& Tikekar, R. 1982, J. Astrophysics Astron. 3, 325 